\title{
Prévention et gestion des dommages dus aux inondations
}

\author{
Jacques Estienne \\ IGPC, Président
}

Pierre-Alain Roche

ICPC, Rapporteur Général

\section{Introduction}

En introduction au présent rapport, le président livre quelques réflexions suggérées par les communications, nombreuses, et les discussions, succinctes, - ceci étant peutêtre la conséquence de cela - ainsi que par sa propre expérience.

Tout d'abord, certains thèmes n'ont guère été évoqués, en dépit de leur importance.

Il est patent que le public ne croit jamais les responsables administratifs lorsqu'ils font état de risques d'inondations.

Ce manque de crédibilité provient en partie - c'est l'argument habituel - de la nature et de l'insuffisance de précision des documents établis à cette fin: les $23^{\mathrm{e}}$ journées ont montré que l'on se préoccupait beaucoup de l'amélioration des méthodes appliquées en la matière et des modes de communication à destination du public, et que les résultats étaient dans l'ensemble, prometteurs et, pour certains, déjà opérationnels. Ces avancées ne seront cependant significatives que si elles sont accompagnées par une augmentation substantielle des financements.

En dehors de raisons inavouables, en tous cas non avouées, telles que la pression de l'urbanisation et le coût du foncier, ce manque de crédibilité tient aussi à l'importance des facteurs psychologiques et sociologiques: l'individu a tendance à vouloir oublier toute catastrophe, c'est un événement exceptionnel qui ne se reproduira plus, il faut tourner la page... et l'on constate que les catastrophes sont ressenties différemment selon les régions. Ces facteurs ne paraissent guère étudiés en comparaison des aspects techniques et pourtant cela permettrait de comprendre ces comportements et d'agir sur eux, avec une efficacité sans doute plus grande que celle des actions strictement techniques, notamment par une politique pertinente de communication, avant et après l'événement, catastrophique ou non.

Les $23^{\mathrm{e}}$ journées ont bien montré la richesse des différents types de modèles hydrologiques, leur quantité, peutêtre moins leurs qualités opérationnelles. En tous cas, la modélisation mathématique des phénomènes est un puissant outil de progrès. On finit cependant par oublier que « la carte n'est pas le territoire».
Aussi faudrait-il donner une plus large place à l'observation et à l'état d'esprit naturalistes, ce qui enrichirait les conceptions issues de la seule modélisation. Un participant aux $23^{\mathrm{e}}$ journées a ainsi noté que l'on n'avait pratiquement pas parlé de géologie...

On a un peu parlé de sciences naturelles et d'écologie, dans le cadre de la question II, beaucoup moins cependant qu'on ne l'avait souhaité au départ. Or c'est fondamental pour l'étude des remèdes contre les inondations, auxquels la loi sur l'eau impose maintenant de prendre en compte les écosystèmes aquatiques.

Il nous semble que, de même qu'elle s'est ouverte au fil des ans, depuis sa création, sur d'autres domaines que l'hydroélectricité, la Société Hydrotechnique de France devrait le faire, et plus résolument, sur les thèmes évoqués ci-dessus, à savoir la sociologie, les sciences naturelles et l'écologie.

Ensuite, les recherches et applications en matière de prévision des pluies et des crues, nombreuses et souvent pertinentes, donnent le sentiment d'une certaine dispersion, malgré les contacts existants.

On a bien constaté que beaucoup de recherches et d'applications pratiques étaient en cours, soit dans les laboratoires universitaires ou du C.N.R.S., soit dans les grands organismes publics, soit dans certains services opérationnels en raison de leur importance ou de leur histoire. On a pu également se réjouir d'un rapprochement incontestable des services de Météo-France et des hydrologues. Des progrès réels sont donc d'ores et déjà accomplis, mais ne faudrait-il pas, et ne pourrait-on pas aller plus loin ?

De même, l'application à un territoire urbanisé ou à un bassin donné de méthodes de prévision performantes, selon les recherches, mériterait d'une part d'être comparée à ce qui se fait ailleurs, d'autre part d'être validée opérationnellement, ce qui ne peut être formellement réalisé qu'après l'expérience de crues ou de pluies constatées, parmi lesquelles doivent figurer des pluies ou des crues non fréquentes. Cela nécessite la mise en oeuvre d'un suivi au niveau national.

On a ainsi esquissé ce qui serait le rôle d'un organisme technique national, chargé plus généralement de formaliser des objectifs de recherches, d'en coordonner et d'en suivre la réalisation, d'assurer les comparaisons entre différentes méthodes et leur validation, de constituer une doctrine et 
de la diffuser, de fournir un appui technique aux services opérationnels. Les missions de cet organisme devraient d'ailleurs s'étendre à tous les problèmes liés aux inondations, notamment l'entretien des rivières.

Enfin, au cours des $23^{\circ}$ journées, on a assez peu évoqué les risques spécifiques susceptibles d'être provoqués par un dépassement des hypothèses faites par les concepteurs (par exemple, crue supérieure à la "crue de projet ») ou par un dysfonctionnement (par exemple, rupture de digues ou interruption de la transmission de données d'observation en temps réel), même si ces aspects n'ont pas été passés sous silence.

Il est cependant indispensable d'imaginer a priori ce que pourra être le phénomène auquel on sera alors confronté, et d'en déduire les solutions à mettre en oeuvre au cours de l'événement, y compris pour l'organisation des secours. Dans cet ordre d'idées, il est nécessaire de distinguer les crues ou pluies fréquentes conduisant, en principe, seulement à des dommages matériels et qui ressortissent à des analyses économiques, à des réalisations d'ouvrages ou de systèmes de prévision, des crues ou pluies exceptionnelles ou brutales susceptibles de provoquer des catastrophes et qui ressortissent à d'autres mesures, notamment en matière de conceptions urbanistiques.

Dans les dysfonctionnements d'ouvrages ou de réseaux de prévision, il faut citer particulièrement le cas des vannes, notamment lorsque leur manoeuvre est automatisée. II est prudent de n'en prévoir que lorsqu'on ne peut pas faire autrement (cas de quartiers anciens d'agglomérations, par exemple) et, alors, d'en renforcer la fiabilité, par exemple en créant des redondances dans le système de transmission.

Dans le même ordre d'idées, il ne faut pas oublier que, souvent, les ouvrages de protection ne sont sollicités que rarement, ce qui implique d'une part qu'ils soient soigneusement entretenus, d'autre part qu'il en soit tenu compte dans leur conception ou, pour les ouvrages anciens, dans leur renforcement: on constate généralement le contraire, par exemple pour les digues.

On pensera peut-être que les réflexions précédentes sont désespérément banales, et pourtant chacun pourra vérifier qu'elles sont la plupart du temps oubliées : on peut citer les ruptures des digues de Camargue et, dans les années récentes, la rupture d'un barrage asiatique par submersion, aucune des 14 vannes d'évacuation des crues n'ayant pu être manoeuvrée.

Compte-tenu du nombre très important de communications, certaines ne sont pas référencées dans ce rapport, qui a tenté d'être synthétique : que les auteurs veuillent bien en excuser le rapporteur général. Et que les lecteurs y trouvent une raison de plus pour se reporter aux actes du colloque.

\section{Suivi et prévision immédiate des pré- cipitations}

\subsection{Le radar}

«Les Américains envisagent [même] d'assurer la mesure quantitative des chutes de pluie au moyen du radar» apprend $\mathrm{M}$. Pierre KoCH dès la troisième page de son cours d'hydrologie à l'ENPC en 1954 [1].

Nous voici quarante ans plus tard. Que lisons-nous sous la plume de M. Kerdoncuff, A. Marie, et F. SEvault
[2]? «Météo-France a entrepris plusieurs études pour valoriser l'information des radars météorologiques : le but ultime est de parvenir à une utilisation quantitative de ces données radar [...]. Les premiers résultats sont encourageants $»$ !

Dès le début des années 1960, FABRET, DUPOUYET et leur équipe, installaient en Dordogne le radar de Grèzes destiné à contribuer à l'annonce des crues. Le pari d'une utilisation qualitative des images radar, comme un outil d'aide à la compréhension de la dynamique des épisodes dont les réseaux de pluviographes au sol ne donnent qu'une représentation très partielle, était rapidement gagné. Mais celui de la quantification de la pluie à partir de ces images est d'une autre difficulté. Malgré ces succès précoces, l'utilisation des radars en hydrologie a ensuite végété assez longtemps.

Depuis 10 à 15 ans, la recherche a redémarré et des études et des thèses s'accumulent dans les laboratoires météorologiques et hydrologiques en France. Des programmes de grande ampleur (North West Weather project en Grande Bretagne, Nexrad aux Etats-Unis) ont été lancés à la même époque et traduisent tout l'intérêt potentiel de cette quantification. Tout ce travail nécessite la validation de longues séries de données. Il suppose aussi une continuité. Quand F. BRESSAND, de la DDE du Gard et A. KAPFER, de RHEA [3] font le bilan de l'apport des données radar de pluie dans ce département, ils se réfêrent à leur expérience personnelle, depuis qu'en 1989 le Ministère de l'Environnement a pu faire bénéficier quelques services d'annonce des crues du déport des images METEOTEL à l'occasion d'une convention qui intégrait le radar de Grèzes dans le réseau ARAMIS et marquait le début de la collaboration. Mais, par ailleurs, D. FAURE, H. ANDRIEU, D. CREUTIN [4] rendent compte de l'opération Cévennes 86-88 financée par le même Ministère de l'Environnement, sur le même territoire, avec des moyens techniques certes différents, mais avec des objectifs généraux proches. Dans un cas on se réfère à 3 événements pluvieux, dans l'autre à 2 épisodes. On ne peut pas dire que l'on croule sous l'information ! Les équipes se connaissent, et ont des critères d' évaluation des résultats qui ne diffèrent pas fondamentalement, mais il reste difficile de faire la synthèse de leurs 2 expériences sur la base de leurs 2 contributions. Prenons les pluies horaires: dans un cas on compare les lames d'eau intégrées sur 3 bassins-versants de 300 à $550 \mathrm{~km}^{2}$. Dans l'autre, on compare des valeurs ponctuelles à partir de pluviographes d'une part, et de données radar calibrées d'autre part (pixel radar d'une part, et pluviographe d'autre part). Des variations d'un facteur 2 apparaissent dans le premier cas, et d'un facteur 10 dans le deuxième.

L'urgence de stabiliser un processus de recherchedéveloppement dans ce domaine est évidente. J.P. DuPOUYET, avec J.J. VIDAL, L. BOURREL et H. SAUVAGEOT [5] l'ont bien compris, en cherchant à établir un projet permettant de capitaliser l'expérience des données radars et pluviographiques sur le bassin supérieur de la Garonne, et, en réunissant autour de ce projet un comité d'experts, d'assurer la circulation de l'information.

La construction d'un tel processus de recherchedéveloppement n'a rien d'une démarche centralisatrice et réductrice des initiatives. Prenons l'exemple de l'opération lancée par M. SOTTY et R. VEYRIER à la Ville de Marseille, et présenté par G. Delrieu, R. Veyrier, M. Queau et D. FAURE [6]. Il s'agit, constatant les insuffisances criantes 
de la couverture ARAMIS dans les villes méditerranéennes où se concentrent des risques hydrologiques majeurs, d'étudier comment compléter au maximum cette information. L'idée d'utiliser un petit radar en bande $\mathrm{X}(\lambda=$ $3,2 \mathrm{~cm}$ ) a mûri quelques années avant que les tests ne s'engagent. Il faut dire que chacun s'y est mis pour dissuader l'équipe de se lancer dans l'opération. En effet, le choix fait depuis longtemps de travailler en bande C $(\lambda=5 \mathrm{~cm})$ ou mieux en bande $S(\lambda=10 \mathrm{~cm})$ s'impose comme une évidence à qui que ce soit qui s'intéresse au sujet : ce n'est qu'à partir d'une longueur d'onde de $5 \mathrm{~cm}$ que l'on commence à pouvoir dominer l'atténuation des ondes par les précipitations, qui «cachent " en quelque sorte les précipitations situées plus loin, tout comme le brouillard diminue la visibilité dans les longueurs d'onde du visible. Ces obstinés ont néanmoins poursuivi leur démarche et c'est tant mieux. Ils ne nous présentent pas encore un diagnostic sur la faisabilité et l'intérêt de poursuivre, mais nous l'attendons avec impatience, tant il est vrai que le progrès pratique et opérationnel ne procède pas toujours d'une application frileuse de principes théoriques.

P. Tourasse, R. Garçon, C. Obled, V. Aubonnet et C. DuFOuR [7] analysent de façon très complète l'épisode du 21-22 septembre 1992, en intégrant, dans une étude a posteriori, l'ensemble des informations disponibles sur cet épisode. On constate tout d'abord qu'en situation de crise une partie du réseau au sol est mise en défaut : 9 des 11 stations du réseau d'EDF de la Haute Loire. Nous reviendrons sur cette question au point 2.3 .

D'autre part, l'analyse d'épisodes majeurs tels que celui-ci ne peut se faire qu'à une échelle très globale : ici, tout le quart Sud-Est de la France, soit plus de 300 pluviographes sont étudiés. Et, bien que les auteurs n'y insistent pas trop, on ne peut que regretter une fois de plus la dispersion de l'archivage des données pluviographiques : est-il bien logique qu'il faille faire le tour d'une vingtaine de services différents pour obtenir de quoi travailler sur un épisode ? Des efforts ont permis de constituer une banque nationale pluviométrique, d'organiser un minimum d'archivage des données radars, mais pour la pluviographie, les études se sont succédées sans qu'aucune décision ne se prenne, et c'est bien dommage.

La cartographie de l'épisode heure par heure montre, à cette échelle globale, les lignes directrices de sa dynamique. En particulier, le développement des orages dans la matinée du 22 septembre, des Alpilles au Ventoux en rive gauche du Rhône, n'est pas lié à un déplacement des foyers cévenols, qui ont perduré, mais à une dynamique locale indépendante et plus complexe.

Les données radar diffusées dès que le radar MELODI de Nîmes n'a plus été en panne (le 22 septembre à 10 h 30) ne bénéficiaient pas des progrès récents faits sur le réseau ARAMIS. On y retrouve donc les défauts, déjà constatés antérieurement, qui ont conduit à l'installation opérationnelle de CALAMAR. Les auteurs constatent sur les données corrigées qu'elles apportent des compléments d'information intéressants, au niveau qualitatif.

L'étude présentée insiste enfin sur l'utilité de la visualisation METEORAGE. En effet, l'intensité de l'activité orageuse est un indicateur complémentaire intéressant. Il doit cependant, et les auteurs en conviennent, être considéré comme une assistance à la compréhension des phénomènes, mais ne peut être considéré isolément.

Au cours du débat, les démarches visant à une validation globale de la chaîne radar-modèle pluie débit ont été discutées : pourquoi ne pas considérer l'ensemble comme un tout, et assurer un calibrage des estimations radars par recalage en fonction de débits observés sur de très petits bassins-versants ?

Cette démarche, qui se veut pragmatique comporte des risques réels, comme l'a souligné C. OBLED : d'une part il ne faut pas oublier que la réflectivité radar n'est pas une mesure de la pluie, et d'autre part on a déjà assez de difficulté avec la globalité de la phase terrestre du cycle de l'eau, pour laquelle on ne dispose pas de beaucoup d'indicateur d'état, pour accepter une dégradation du sens physique de l'entrée pluie et de supprimer le point de contrôle indispensable que cela constitue, même si on connaît les imperfections de la mesure de la pluie au sol.

En résumé :

- au plan opérationnel strict, le développement d'ARAMIS et du déport des images METEOTEL dans les services d'annonce de crues est d'ores et déjà un succès, qu'il faut conforter en complétant la couverture radar du Sud-Est de la France au plus vite. En effet, les images radar donnent une compréhension de la dynamique des épisodes précipitants qui est indispensable. Ceci ne dispense en rien de réseaux pluviographiques denses, correctement gérés et entretenus, et dont les données soient critiquées, validées et archivées :

- nous constatons les premiers progrès liés au développement des relations entre les hydrologues et les météorologues, auxquelles on travaille depuis de nombreuses années. Les conditions sont aujourd'hui réunies pour leur intensification:

- avec le réseau ARAMIS, l'hydrologie et la météorologie radar commencent à disposer des bases de données nécessaires pour sortir du bricolage dans lequel elles étaient confinées jusqu'à présent. De nombreux travaux existent, que l'on peut valoriser en les confrontant à l'expérience de ces données:

- c'est maintenant qu'une structuration des équipes de recherche-développement autour de quelques sites radars à vocation mixte opérationnelle et de recherche apporterait les meilleurs progrès. en développant une pluridisciplinarité hydro-météorologique qui a fait des progrès, mais reste encore balbutiante:

- l'archivage et la disponibilité des données pluviographiques est toujours une difficulté non résolue.

\subsection{La prévision immédiate}

La prévision immédiate des précipitations est, à l'évidence, l'unique chance de débloquer les limites actuelles de la prévision des crues en temps réel pour les petits bassinsversants. Dans un rapport de 1989, [8] puis au colloque de Nainville-lès-Roches, [9] en 1992, nous insistions sur le fait que c'était là qu'il convenait de faire porter prioritairement les efforts.

J.D. CREUTIN, H. ANDRIEU, ont collaboré avec W. KRAJEWSKI, de I'Université d'Iowa, pour nous présenter aujourd' hui une synthèse des perspectives dans ce domaine [10].

Les modèles déterministes procèdent d'une réduction progressive des mailles des modèles météorologiques de circulation générale. L'objectif d'atteindre une maille de $10 \mathrm{~km}^{2}$, qui parait un minimum pour atteindre un objectif opérationnel, est ambitieux. Mais il ne s'agit pas que de cela. Il faut également y intégrer une représentation per- 
tinente des processus directement impliqués dans la généralisation des précipitations. J.P. BOURDETTE, dans les actes du colloque de Nainville [9], décrivait fort bien les démarches en cours à la Météorologie Nationale pour aller dans ce sens. Il faudra pouvoir s'affranchir de l'hypothèse hydrostatique de répartition des pressions des modèles opérationnels actuels puisque les mouvements de convection jouent un rôle essentiel dans les processus en jeu au sein des cellules précipitantes. De même une représentation des 2 phases liquide et vapeur de l'eau et de leurs échanges est indispensable. C'est là un programme lourd de recherche qui bénéficie de nombreux travaux partiels déjà réalisés, mais suppose la mise en oeuvre de très importants moyens, tant numériques que d'instrumentation.

Les modèles d'advection se sont développés avec les données radars. En cherchant à prévoir au mieux le déplacement des cellules précipitantes observées, leur champ est naturellement cantonné aux très courtes échéances et aux faibles échelles d'espaces, ce qui en fait un outil privilégié de l'hydrologie urbaine.

Une «troisième voie» nous est présentée, pour la première fois sur la base de résultats concrets, par H. ANDRIEU et V. THAUVIN en collaboration avec M. FRENCH de l'université de Louisville, aux USA [11]. Il s'agit, en s'inspirant de travaux de GEORGAKAKOS et BRAS datant d'une dizaine d'années, d'utiliser les données radar à chaque pas de temps pour identifier la colonne atmosphérique précipitante et en quantifier le contenu en eau précipitante, des données météorologiques au sol donnant par ailleurs une indication du flux d'air humide entrant dans la colonne.

Bien sûr, les résultats obtenus sont partiels. Mais l'analyse et l'interprétation qui en sont donnés montrent qu'il y a là une voie prometteuse.

En résumé :

- la prévision à très court terme est un passage obligé aujourd'hui pour progresser sur la gestion des conséquences des épisodes précipitants, dont la stationnarité ou les déplacements, ainsi que la persistance, conditionnent l'essentiel des choix opérationnels dans l'organisation des secours :

- il y a plusieurs voies, pour avancer dans ce domaine, qui méritent d'être défrichées au plus vite.

\section{Prévision des crues}

L'organisation d'une prévision des crues performante repose sur deux composantes essentielles :

- les moyens rapides et fiables de collecter des informations pertinentes ;

- l'élaboration d'une prévision en temps réel par un opérateur généralement assisté par des modèles ou autres moyens d'exploitation de ces informations ;

L'expérience montre que les outils d'intégration de ces 2 composantes en un tout cohérent jouent un rôle essentiel.

\subsection{Les moyens de collecte}

M. Odier et C. Godon [12] tracent un tableau complet de l'évolution des moyens mobilisés par le Ministère de l'Environnement dans ce domaine. Outre le développement des moyens météorologiques dont on a largement parlé cidessus, des moyens nouveaux sont décidés pour relancer la modernisation des réseaux de collecte. D'abord équipés au coup par coup sur la base de matériels disparates et généralement très coûteux, ces réseaux ont ensuite été équipés, en s'inspirant d'ailleurs des méthodes plus professionnelles de la Météorologie Nationale et d'EDF, d'un produit standard établi sur la base d'un cahier des charges national établi avec l'ensemble des services concernés, et faisant l'objet du développement d'un prototype soumis à des tests rigoureux. C'est la gamme NOE, qui a rapidement évolué, notamment au niveau des logiciels des postes de collecte. En effet, les développements d'outils standard ont permis de substituer aux premières boîtes à outils développées spécifiquement pour cette application des progiciels plus riches et apportant une compatibilité meilleure avec l'environnement. Il convient de souligner l'aspect exemplaire de ce travail d'adaptations successives, épousant l'évolution des techniques, et fondé sur une réflexion collective des utilisateurs, qui aura assuré une pérennité de 10 à 15 ans au concept initial, ce qui est tout à fait satisfaisant au plan économique.

Il est temps maintenant d'assurer la relève. C'est ce à quoi s'emploie un groupe qui définit les spécifications d'un nouvel équipement, la station de l'an 2000, baptisée P.L.Q 2000.

Le développement des réseaux d'annonce des crues n'a cependant pas été aussi rapide que l'on aurait pu espérer : seulement 700 stations sont aujourd'hui équipées. Les raisons en sont multiples. Jusqu'à une date récente, les crédits tant d'investissement que de fonctionnement, étaient retombés à un niveau bas. Par ailleurs, le principe de cofinancement avec les collectivités pose toujours difficulté dans certains départements, et enfin l'aptitude des services d'annonce des crues à monter un projet est parfois limitée.

Mme GODON et M. ODIER mettent par ailleurs à juste titre l'accent sur la fiabilité des réseaux. Depuis que ces réseaux sont en service, il est frappant qu'il y ait aussi peu de compte-rendu de leur fonctionnement et d'analyse de leur fiabilité. Il est certain que l'on ressent ici la faible implication d'un réseau technique structuré ce qui est préoccupant pour l'avenir. Cela donne d'autant plus d'intérêt à la contribution de L. Moulin et J. REINBOLT [13] sur le réseau de la Loire.

En effet on y présente une bonne analyse de fiabilité du réseau de la Loire, notamment, et c'est ce qui manque souvent, en distinguant les périodes d'orages et d'inondation de celles de fonctionnement courant. Si les taux globaux peuvent apparaître acceptables $(90 \%$ à $94 \%$ de bon fonctionnement) en revanche, en situation de crise comme lors de l'épisode du 21 et du 22 septembre 1992 et malgré un réel effort d'intervention, on est encore à un taux de défaillance de $25 \%$.

Les auteurs proposent, en l'étayant, un doublement des systèmes sur les sites sensibles, en précisant bien qu'il faut doubler l'ensemble de la chaîne, et non seulement soit le capteur, soit le vecteur de transmission contrairement à ce que nous avions pu préconiser il y a quelques années dans un souci d'économie.

Le même épisode du 21-22 septembre 1992 a fait l'objet d'une étude approfondie de la part de C. LALLEMENT et de P. TOURASSE [14] à partir du réseau EDF, sur 34 stations des hauts-bassins de l'Ardèche, de la Loire, de l'Eyrieux et de la Cèze. Durant l'épisode, $44 \%$ de ces stations ont été partiellement ou totalement en défaut. Les défauts de 
protection de l'alimentation ou de la ligne P.T.T représentent à eux seuls $30 \%$ de ces défaillances.

Vis-à-vis de ces défauts, les auteurs présentent la démarche d'étude et de qualification des matériels mise en place par EDF : selon leur analyse, il s'agit plutôt d'une vulnérabilité liée au site. Des efforts de durcissement partiels se sont révélés inopérants : il faut revoir entièrement les stations quand elles ont été touchées.

Les problèmes de liaison téléphonique et de capteurs sont également étudiés en détail.

Les auteurs insistent enfin, et comment ne pas les rejoindre, sur l'importance de disposer en temps réel de sources d'informations variées et rigoureusement indépendantes.

J.L RoY, [15] à partir de son expérience sur le même bassin, s'interroge sur les principes de l'organisation de l'annonce des crues mise en place en 1984. Il remarque en effet que la réforme de 1984 , pour clarifier les rôles et les responsabilités, a introduit une chaîne d'intervenants : service d'annonce des crues, Préfet, Maires, services de secours, qui peut présenter une certaine rigidité. Il propose donc que ce service réglementaire soit doublé par un système direct allant du service d'annonce des crues aux maires, grâce au développement de matériels permettant la diffusion automatique simultanée de messages à de nombreux destinataires. Il évalue le gain de temps à près d'une heure dans certains cas.

Cette suggestion devrait retenir toute l'attention des autorités, malgré les quelques difficultés de principe qu'elle semble a priori poser, mais qui ne sont qu'apparentes. Mme Godon et M. OdiER évoquent d'ailleurs des perspectives positives dans ce sens.

Les collectivités locales peuvent également être présentes de façon autonome dans les systèmes de collecte de données. A.P. Bouillot, B. Voron et M. MelKI [16], après nous avoir rappelé quelques caractéristiques de l'épisode bien connu du 3 octobre 1988, nous présentent le réseau mis en place à Nîmes comportant 8 pluviomètres et 10 limnimètres, utilisant le système NOE, avec pour vecteurs de transmission la radio et le téléphone.

L'intérêt particulier de cette expérience est le couplage qui existe entre les réseaux d'annonce des crues de la DDE et de la Ville de Nîmes dont les postes centraux peuvent accéder aux mêmes stations, et échanger directement des informations collectives. Il semble que le partage, entre les 2 services, des responsabilités d'alerte opérationnelle soit assez bien précisé.

En résumé :

- les réseaux de collecte automatique ont marqué le pas ces dernières années. Les annonces faites d'une relance dans ce domaine sont encourageantes;

- la fiabilité opérationnelle de ces systèmes, encore relativement récents (15 ans pour les plus anciens) reste à améliorer, pour les situations de crise auxquelles ils sont destinés, même si dans l'ensemble ils fonctionnent de façon plutôt satisfaisante. Les études, dans ce domaine, sont encore insuffisamment nombreuses ;

- le système réglementaire de l'annonce des crues mis en place en 1984 mérite aujourd'hui, pour la diffusion de l'information aux maires, d'être réétudié ;

- aucun développement de l'annonce des crues par les services de l'Etat dans les bassins-versants non équipés, correspondant à des délais d'intervention de 4 à 6 heures, ne semble envisagé. Pourtant des besoins existent, notamment en amont d'agglomérations de taille moyenne à petite;

- la limite du partage des responsabilités entre les villes et les services de l'Etat reste souvent ambigüe.

\subsection{Les modèles de prévision des crues}

C. Obled, J.Y. Rodriguez et G.M. Saulnier [17] présentent une synthèse de la démarche d'ensemble qu'ils proposent d'adopter pour les études de prévision des crues sur les bassins-versants de faible taille (10 à $1000 \mathrm{~km}^{2}$ ) soumis à des crues-éclairs en utilisant la méthode de la D.P.F.T. On ne saurait trop recommander à chacun la lecture complète de cet article. En effet, il poursuit le style que nous avions cherché collectivement à développer à travers le guide de prévision des crues en 1987 [18]: prendre la démarche d'élaboration d'une prévision à travers la chaîne des réflexions qu'un praticien doit engager, en respectant le principe d'une compréhension progressive des choses : inutile de se lancer à corps perdu dans une procédure complexe et un raffinement particulier, tant que les éléments de base ne sont pas en place. Cette logique, qui paraît évidente, est indispensable : en effet, les publications habituelles ont soit un caractère monographique très particulier, soit un degré de spécialisation technique très prononcé, qui occultent le savoir-faire pratique.

Quelques préconisations de ces auteurs paraissent essentielles :

- Recueillir les données au pas de temps le plus faible possible, 1 heure au minimum pour les petits bassinsversants. Cela veut en particulier dire qu'en l'absence de telles données, il y a une première décision qui s'impose : équiper le bassin-versant, en ayant à l'esprit que la densité de postes pluviographiques à atteindre est au minimum de un poste pour $100 \mathrm{~km}^{2}$ pour un pas de temps horaire.

Faire très attention aux données de débit: on a l'impression de toujours se répéter, mais il ne faut jamais perdre de vue que l'essentiel est là.

- Analyser sommairement la réponse du bassin-versant, pour se faire une idée des temps de réponse. C'est indispensable pour faire un premier diagnostic de faisabilité : les délais sont-ils suffisants pour envisager quelque chose ?

C. Obled, J.Y. Rodriguez, et G.M. Saulnier proposent pour cela d'utiliser une identification simple de la D.P.F.T, à condition de disposer d'une quinzaine de crues. Sur moins d'épisodes, ce qui est un cas courant, il convient de rappeler que les méthodes manuelles restent pertinentes.

- Analyser ensuite la variabilité du comportement du bassin-versant.

Les auteurs proposent d'approfondir, en considérant que la fonction de transfert peut être variable, par exemple dans une logique de zones contributives où les zones saturées sont susceptibles d'évoluer en début d'épisode. Sur ce point nos avis divergent, bien que l'on retrouve chez ces auteurs une modération de bon aloi lorsqu'ils indiquent « on préférera retenir une fonction de transfert unique ». Il me semble en effet, à ce stade d'étude, préférable d'abandonner la démarche sur les fonctions de transfert, qu'il me paraît inutile de peaufiner, mais de passer à une représentation globale production-transfert, par un modèle le plus simple possible. 
- Dans l'esprit de la D.P.F.T les auteurs décrivent ensuite le choix du modèle de production. Il conviendrait d'élargir cette réflexion au choix du modèle global, car la séparation de la production et du transfert est artificielle, notamment en période de crue.

Ils privilégient a priori une modélisation des zones contributives pour les bassins à forte couverture végétale et suggèrent d'en rester à une vision hortonienne pour des bassins très dénudés ou partiellement urbanisés. C'est de bon sens, même l'on ne dispose pas vraiment d'une preuve étayant ces recommandations.

- Les auteurs insistent sur l'intérêt de rester à une représentation non distribuée tant du point de vue de la réponse des composantes du bassin que de la répartition spatiale de la pluie, tant que les données dont on dispose ne le justifient pas expressément.

Ce point parait devoir être souligné. Les auteurs ont en effet sur ce domaine une expérience considérable, et ont fait suffisamment de tentatives dans le sens de la distribution spatiale pour que leur conseil soit écouté avec la plus grande attention.

- Concernant la mise en cuvre opérationnelle, nos conclusions se rejoignent également : mais nous y reviendrons après avoir examiné les autres contributions.

X.L. YANG, C. MiChel et P.A. RoCHE [19] présentent une recherche sur les modalités de recalage d'un modèle pluie-débit conceptuel en temps réel. Cette question apparaissait en impasse il y a quelques années. En effet, de nombreux travaux introduisaient des techniques de traitement des écarts en aval des modèles, ou des procédures de filtrage des paramètres ou des niveaux des réservoirs des modèles qui ne donnaient guère satisfaction en réalité, et l'opération d'inter comparaison des modèles de prévision que l'Organisation Météorologique Mondiale avait organisée en 1990 à Vancouver avait à juste titre mis un coup d'arrêt à ces errements [20].

L'initiative présentée ici veut tirer les enseignements de ces échecs, et recherche un mode d'adaptation des modèles pluies-débits conceptuels qui préserve la structure du modèle, en corrigeant les paramètres et les niveaux, pour assurer la préservation des volumes écoulés. Par ailleurs, un système de durée rétroactive d'ajustement, sur un nombre suffisant de pas de temps, assure que le modèle, lors de l'émission de la prévision, ne soit pas dans un état destructuré par un recalage aveugle au fur et à mesure. On recherche ainsi en temps réel des valeurs des paramètres qui soient les plus proches possible des paramètres d'un calage historique, tout en assurant une représentation cohérente des processus constatés sur les derniers pas de temps.

Cette communication réouvre par ailleurs un débat autour du choix des modèles pour la prévision. Deux approches sont en effet traditionnellement en présence :

La première approche, que ce travail contribue à alimenter, suppose qu'il est intéressant de faire fonctionner un modèle pluie-débit en continu représentant l'ensemble du cycle de l'eau sur le bassin-versant.

La deuxième approche, qui est soutenue notamment par l'expérience opérationnelle acquise par la D.P.F.T développée par D. DUBAND à l'Electricité de France, consiste à mettre au point un modèle spécifique aux situations de crues, dit « modèle d'événement ", de façon économe dans la paramétrisation, d'un état initial représentatif du bassinversant.
Au bénéfice de la deuxième approche, la possibilité de privilégier la représentation du fonctionnement de crue, les modèles globaux pluie-débit étant, dans leur calage, parfois mieux adaptés à la représentation des écoulements de base qu'à celui des crues.

En résumé :

- la recherche en matière de prévision des crues paraît encore bien dispersée et artisanale. Des options importantes pour mettre en oeuvre des modèles opérationnels (modèles continus et/ou modèles à événements) sont encore largement incertaines;

- une initiative nationale, sous la forme d'un travail rigoureux d'intercomparaison, suivant un protocole clairement défini, serait nécessaire pour avancer ;

- la diffusion des méthodes de prévision au sein des services d'annonce des crues est encore très faible. Alors que dès 1854 BELGRAND mettait en auvre des méthodes très élaborées de propagation des crues au service de navigation de la Seine, il semble qu'en 1994 les services d'annonce des crues n'aient, pour beaucoup d'entre eux, ni la compétence technique ni la disponibilité, pour utiliser des méthodes modernes de prévision, et que l'écart entre la recherche et l'application ne se réduise pas autant qu'il serait souhaitable. L'absence de communications de services opérationnels, qui traditionnellement, sur la base de leur expérience de terrain, étaient les promoteurs de progrès, est de ce point de vue inquiétante.

\subsection{Le développement de systèmes multi-modèles ou multi- experts pour le temps réel}

L'expérience opérationnelle montre que l'effort de développement d'un système de prévision des crues n'est pas terminé lorsque l'on dispose d'une part d'un réseau de collecte de données, et d'autre part de méthodes de prévision des crues.

En effet, l'intégration de l'ensemble pose un grand nombre de difficultés qu'il ne faut pas sous-estimer. Le nombre des contributions sur ce thème à ces journées est sans aucun doute l'une des originalités et des meilleures preuves de la maturité de l'hydrologie opérationnelle au sein de certains services.

D. Duband, J.L. NÈgre, J.A. Cunge et A. Kapfer [21] présentent un projet intéressant en ce sens. Il s'agit de capitaliser un ensemble d'expériences françaises par l'élaboration et la validation d'un système destiné ensuite à une large diffusion.

D'une part, nous l'avons vu, il y a nécessité d'intégrer, pour ce qu'elles sont, les données radar, et de les valoriser autant que possible.

D'autre part, la multiplicité des informations collectées sur un système en temps réel permet de nombreuses combinaisons représentant autant de modélisations possibles. C'est d'autant plus intéressant que ces informations restent toujours des représentations partielles de la réalité physique, qui peuvent se trouver en défaut dans un contexte opérationnel déterminé. Le recours à une procédure multimodèles ou multi-experts développée par le CERGRENE en 1987 [22] bénéficie maintenant d'une bonne expérience de terrain sur le bassin de la Garonne; de même, on connaît bien maintenant le fonctionnement opérationnel de la D.P.F.T déjà citée. Le projet MISTERE présenté intègre tout cela. Il conviendrait que des modèles pluies-débits en continu soient également intégrés à ce processus. En tout 
état de cause, l'intégration de tous ces éléments, selon un protocole rigoureux, sera sans aucun doute une étape décisive, aboutissement de cette première génération de développement des outils pour en permettre la mise à disposition du plus grand nombre des services et en assurer les nécessaires validations par l'emploi opérationnel.

Mais il faut aussi préparer les outils de la deuxième génération.

L'initiative de J.P. DupouYet, P. COHEN, C. Feliu, P. GLIZE et J.J. VIDAL [23], préparée depuis bientôt 2 ans, d'élaboration du «SEC » (système multi-expert pour la prévision des crues) va dans ce sens.

Ayant maintenant l'expérience du succès de la procédure multi-modèle et du système «diagnostic-pronostic » du CERGRENE, les auteurs passent à l'étape de la formalisation de l'expertise au sein d'une architecture de traitement délibérément tournée vers la valorisation de la plusvalue apportée par le prévisionniste.

Il s'agit de faciliter:

- l'élaboration de diagnostics, globaux ou particuliers, en situation de crise :

- d'apporter les éléments nécessaires à une anticipation permettant d'agir à temps: il s'agit non seulement de prévoir l'évolution du processus hydrologique, mais aussi de planifier des actions opérationnelles et d'en évaluer la pertinence :

- de former des débutants, et même des agents expérimentés, par l'analyse qui peut être faite a posteriori des événements vécus.

Cette démarche est en plein développement.

C. Thirriot et H. HABAiEB [24] vont dans le même sens. L'idée d'expérimenter avec un groupe de "prévisionnistes " de divers horizons la mise en oeuvre de prévisions manuelles recentre, elle aussi, le débat sur le comportement et les performances du prévisionniste, l'outil de prévision étant ramené à sa juste valeur d'accessoire. Même si un tel travail ne permet pas encore directement la mise au point des aides utiles au prévisionniste, il a la grande qualité d'ouvrir un champ nouveau de réflexion, en rompant avec les études traditionnelles.

En résumé :

- un système tel que le projet MISTERE, dûment complété pour capitaliser toutes les expériences acquises sous une forme accessible à tous les services d'annonce des crues, est une urgence. Il faut espérer que les décisions, favorables dans leur principe soient rapidement traduites dans les faits, et que les services opérationnels soient effectivement associés à toutes les phases du développement de ce qui devrait pouvoir constituer rapidement leur outil ;

- les perspectives ouvertes par les systèmes-experts sont un prolongement nécessaire de cette première génération d'outils, en recentrant la démarche autour du prévisionniste. Il faudra compter avec plusieurs années d'investissement dans cette voie avant qu'elle ne porte véritablement ses fruits.

\section{Gestion des ouvrages en milieu urbain et ges- tion des aménagements hydrauliques en temps de crues}

J.L. Rahuel, J.L. Negre et J.A. Cunge [25] nous présentent le système de gestion prévisionnelle des inonda- tions qu'ils ont développé pour la ville de Shanghaï, qui avec ses 13 millions d'habitants est le premier centre économique de la Chine, mais est menacé à la fois par les crues du Huang- $\mathrm{Pu}$, et par les raz-de-marée liés aux typhons. Et comme le niveau du sol s'est abaissé de $1,70 \mathrm{~m}$ dans la plaine du fait des pompages de ces 50 dernières années, on comprend l'ampleur des problèmes. Le système intégré de prévision décrit par ses auteurs réunit donc des modélisations des relations pluies-débit, et des trajectoires de typhons, des calculs de cotes de la mer en situation de typhons (intégrant les surcotes liées au vent et à la chute de la pression atmosphérique) et un modèle de propagation hydraulique. Ce système intégré sera bientôt complété par un système d'aide à la décision.

La réunion de l'expérience de la gestion des réseaux d'assainissement ou d'eaux pluviales, d'un côté, et des ouvrages en rivière d'autre part est particulièrement intéressante.

Nous sommes ici dans des domaines qui disposent d'une expérience de plusieurs dizaines d'années. La nature des questions qui sont abordées s'affinent et se complexifient petit à petit, et les objectifs deviennent de plus en plus ambitieux.

Mais si l'on y regarde bien, d'un côté il y a quelques collectivités qui, comme le département de Seine-SaintDenis, et la Communauté Urbaine de Bordeaux, nous alimentent régulièrement des progrès qu'ils font, et d'un autre côté, la grande majorité qui n'a pas bougé véritablement depuis 20 à 30 ans. L'affaiblissement des structures de conseil ou de coordination qui a pu intervenir dans ces domaines suite à la décentralisation rend peu probable que ces disparités diminuent dans les années à venir. Il y a de quoi rester inquiet pour l'avenir.

Les développements récents des systèmes de télécontrôle de la C.U.B nous sont présentés par D. FrÉsIERS et P. Bourgogne [26], et ceux du département de SeineSaint-Denis par J.M. Delattre, G. Auriaux et P. VoIGNIER [27]. Dans les 2 cas, disposant d'un système de télémesure et de télécommande bien rôdé, ayant mis au point les modèles de simulation hydraulique nécessaires, ayant, en Seine-Saint-Denis, beaucoup travaillé à l'optimisation des modalités de gestion des différents ouvrages réglables, les techniciens sont confrontés à la difficulté de l'élaboration d'une interface-utilisateur qui permettra, en temps réel, de tirer les meilleures ressources du système. On retrouve ici les mêmes préoccupations que pour la prévision des crues, avec même une complexité, une multiplicité d'informations et une rapidité nécessaire aux décisions, bien supérieures.

A la CUB, c'est un système-expert qui a été développé pour assurer l'aide à la gestion des situations de crise dans le département de Seine-Saint-Denis, la détection d'anomalies de fonctionnement hydraulique, dont la sensibilité est réglable en fonction de la qualité d'évaluation de la pluie entrante et de la qualité de la modélisation permet de guider un ensemble d'actes "réflexes" de l'opérateur, après qu'un système-expert a fourni une analyse des causes vraisemblables de ces anomalies, sur la base de règles logiques. Par ailleurs, un très grand soin est apporté à la gestion des interfaces graphiques et des modalités d'accès aux bases de données.

Autant dire que l'on voit aujourd'hui, proches d'être opérationnels, des systèmes dont les principes ont été rêvés voilà 10 à 15 ans, alors même que nous n'en avions pas encore les moyens techniques. 
Ces réflexions sont à rapprocher de celles de X. CAlLMAIL et P. Le Delliou [28] sur la prise en compte des situations exceptionnelles et l'élaboration des consignes de crues pour les barrages-réservoirs manoeuvrables.

Il s'agit, dans ce cas, de fournir à l'exploitant les moyens d'une prise de décision, dans des situations peu probables, mais dont les enjeux, en matière de sécurité, sont majeurs.

Tout d'abord, la gestion d'une crue, même si tout se passe bien, est une question complexe, où des objectifs contractoires peuvent intervenir. Il s'agit de concilier, en particulier, le besoin immédiat d'écrêtement de la crue, et le souci d'éviter un remplissage trop précoce de l'ouvrage rendant inopérant le réservoir pour un épisode qui succéderait au premier. Il convient également de ne pas trop vider le réservoir lorsqu'il a un objectif de soutien d'étiage, ou de production hydroélectrique, mais il peut être utile cependant de procéder à une chasse des sédiments.

Dès le début de l'épisode, les organes de contrôle (vannes, etc...) doivent être manoeuvrés, afin d'en vérifier le bon fonctionnement.

Dès que la crue est d'une ampleur exceptionnelle, ou que les évacuateurs sont bloqués, ou qu'on ne dispose plus d'énergie pour les manoeuvres, en bref dès qu'un incident non gérable par les procédures normales intervient, il s'agit d'apprécier s'il y a opportunité ou non de déclencher un plan d'alerte. Pour cela, il faut analyser les risques, et évaluer les délais disponibles, en particulier jusqu'à l'atteinte de la cote de sûreté. Le rôle de l'exploitant est alors crucial et X. CAILMAIL et P. Delliou décrivent les aides mises à sa disposition.

En résumé :

- la tendance que nous constations sur les systèmes de prévision de crue de développer des systèmes-experts tournés vers l'assistance au prévisionniste est déjà largement engagée et opérationnelle dans les domaines où il y a manoeuvres d'ouvrages en situation de crise, que ce soit dans le secteur urbain ou sur les grands barrages;

- le diagnostic continu de la situation, y compris des défaillances, l'établissement de règles de comportement tournées vers l'analyse permanente des délais disponibles pour intervenir, sont des points de passage obligés d'un tel système d'aide à la décision :

- les développements évoqués ci-dessus restent cantonnés à un trop faible nombre de maîtres d'ouvrage. et il est inquiétant de voir de nombreuses collectivités locales rester dans ce domaine à un niveau très faible. même dans la simple connaissance de leurs réseaux et des événements hydrologiques auxquels elles peuvent être confrontées.

\section{Prévention, communication et système de crise}

On quitte maintenant le domaine de l'opérationnel en temps de crise pour examiner l'évolution, très riche ces derniers mois, des stratégies de prévention.

\subsection{Cartographie}

Une politique de prévention et d'information suppose d'abord que l'on connaisse la nature des risques. Si les études qui ont pu être menées dans les zones couvertes par les Plans d'Exposition aux Risques (P.E.R.) ont permis une analyse précise des territoires concernés, y compris par une modélisation des débordements en zone inondable pour des événements de fréquence de retour déterminée, cela n'a pu concerner en 10 ans qu'un peu plus de 500 communes. C'est un résultat somme toute non négligeable, mais qui est en-dessous des prévisions initiales $(2000$ communes avaient été répertoriées comme prioritaires), et dont le coût aura été important.

La conséquence en était, jusqu'à une date récente, que l'on ne trouvait d'information sérieuse que dans les grandes vallées alluviales, et que, par exemple, les petits bassins-versants montagneux du Sud de la France n'étaient que partiellement couverts, alors que chacun sait que les risques y sont considérables, même s'ils apparaissent diffus sur un très grand chevelu de petits cours d'eau.

A l'occasion du rapport «suivi et prévision des précipitations intenses " [8], J.D. CREUTIN et A. ANDRIEU avaient en 1990 une première carte à très grande échelle (1/5000 000) résultant d'un premier croisement entre risques de précipitations intenses, et rapidité de la réponse des bassins-versants, pour attirer l'attention des pouvoirs publics sur l'intérêt d'engager un recensement de ces secteurs en privilégiant l'exhaustivité par rapport à la précision. Nous renouvellions la recommandation au Colloque de Nainville-lès-Roche en 1992 [9]. Il a fallu attendre les crues de 1992-1993 pour que le programme s'engage. C'est dire tout ce que nous attendons de l'étude présentée par A. Faure-Soulet, J. Lavabre, O. Gilard et P. GiVONE [29] sur l'identification des zones urbaines soumises à des précipitations de type méditerranéen. Cette étude de prédiagnostic porte sur 24 départements.

On bute bien entendu sur des difficultés techniques et il faut mettre des limites. En dessous de $30 \mathrm{~km}^{2}$, on a des difficultés à identifier un réseau de drainage autour duquel on puisse organiser une cartographie : on est alors dans le domaine du ruissellement urbain ou périurbain, qui mérite une attention particulière et des études approfondies.

De même, il faut pour les bassins retenus, renoncer au niveau du prédiagnostic, à une étude hydrologique fine. Trois niveaux d'aléas (courant, rare et exceptionnel) sont proposés pour simplifier. Pour chaque niveau, une formule régionalisée de type CRUPEDIX est utilisée. Du point de vue hydraulique et géomorphologique, on intègre les informations sommaires permettant d'évaluer grossièrement la zone de débordement, à une échelle du $1 / 25000^{\mathrm{c}}$. Enfin, une recherche historique est systématiquement réalisée.

La présentation a permis d'apporter des éléments complémentaires très intéressants. En effet, les auteurs ont eu l'honnêteté de chercher, sur la base de cas bien connus, à cerner les limites de la méthode.

Indéniablement, il existe quelques cas où la méthode est en défaillance n'identifiant pas des risques là où des catastrophes ont pu se produire.

Rien d'étonnant, pour une démarche lancée dans un délai court, et dont l'objectif était de défricher un domaine mal cerné. Des perfectionnements sont certainement possibles. Mais l'essentiel est bien de partir d'une analyse géomorphologique, hydrologique et hydraulique bien cadrée, et de ne plus se fier simplement au recensement par voie de circulaire administrative, où cette identification est faite sans méthode et sans support technique.

Bien différente par ses moyens et ses objectifs est la cartographie réalisée à l'occasion de la mise en oeuvre de 
la démarche inondabilité. Mais on le verra, et cela n'est pas un hasard, il y a quelques points communs.

B. Chastan, O. Gilard, P. Givone et G. Oberlin [30] nous présentent cette démarche dans ses perspectives générales. De quoi s'agit-il ?

On définit d'abord une variable synthétique, baptisée T.O.P. qui puisse servir de support technique à l'ensemble du débat socioéconomique autour de la vulnérabilité aux inondations : cette variable peut être cartographiée, et la discussion peut alors se développer par la comparaison entre sa valeur actuelle et la valeur désirée par chacun des acteurs du débat.

Il s'agit ainsi de favoriser un débat autour des objectifs de protection, au sein d'un groupement des intérêts à l'échelle d'un bassin-versant, et de passer de la sorte d'une conception qui privilégie la réglementation fondée sur des principes d'unicité de la règle et d'équité, à une conception qui privilégie le choix global et la gestion collective de la répartition des risques à des niveaux jugés acceptables, fondée sur une prise en charge des problèmes et des enjeux par les acteurs locaux.

Cette tendance est en bonne adéquation avec certains des axes de la politique que souhaite mettre en place le gouvernement, et sur laquelle nous reviendrons.

O. Barozet, O. Gilard, P. Givone et M. SOMMIER [31] présentent la mise en œuvre de cette méthode pour la constitution d'un véritable outil de gestion quantitative des eaux d'un bassin-versant, celui de la Bourbre. Comme ils le font à juste titre remarquer, l'un des préalables pour une telle démarche est l'existence d'une institution géographiquement adaptée qui soit à la fois en charge des études et des travaux. Ce bassin de $700 \mathrm{~km}^{2}$, regroupant 70 communes, est soumis au double enjeu des zones inondables importantes et d'une forte pression foncière liée à un développement régional dynamique. L'étude a mis en évidence que les développements de l'urbanisation étaient susceptibles de générer des difficultés réelles sur des lieux antérieurement urbanisés : elle a permis de promouvoir une nouvelle politique d'aménagement du territoire concerné fondée sur la mise en réserve d'importantes surfaces pour permettre l'expansion des crues. Les auteurs présentent l'ensemble de l'outil de simulation qui permet de tester divers scénarios d'aménagements, en regrettant pour l'instant son manque de convivialité.

J.N. GAUTHIER et L. GAZULL [32] nous font part du point de vue d'un bureau d'étude n'ayant pas participé au développement de cette méthode, mais qui a choisi de la mettre en oeuvre. Ils insistent sur quelques points

- la légitimité des intervenants : la demande sociale s'exprimant par diverses voix à diverses échelles géographiques, quelle est l'entité qui bénéficie d'une légitimité particulière pour assurer la maîtrise d'ouvrage de la réflexion? On retrouve ici la nécessité à la fois d'une structuration institutionnelle à l'échelle des bassinsversants, mais aussi de groupes de suivi, lors de l'élaboration des schémas, qui intègrent les demandes ponctuelles :

- la précision des résultats : il leur semble qu'une échelle de $1 / 5000^{\mathrm{C}}$ soit un minimum pour caractériser l'aléa ;

- le rôle du bureau d'études, qu'ils voient volontiers comme un animateur des débats, comme un conciliateur, et non plus seulement comme un organe technique du maître d'ouvrage.
H. DUMAY [33] nous présente le produit CALYPSEAU qui permet une représentation des aléas dont une originalité, outre la qualité de l'interface avec l'utilisateur et la qualité des outils cartographiques, est de permettre la visualisation du déroulement d'une crue. De telles visualisations dynamiques avaient été utilisées il y a quelques années, notamment pour les crues de l'Arno à Florence. Elles constituent sans nul doute un outil de pédagogie et de sensibilisation tout-à-fait intéressant.

CALYPSEAU est également un outil qui, fondé sur un système d'informations géographiques, permet une représentation fine de l'aléa et de la vulnérabilité des biens exposés.

L'idée de l'auteur d'utiliser de tels outils dans le cadre de l'instruction des permis de construire, ou à l'occasion des groupes de travail de POS est séduisante, parce qu'elle montre que l'on peut dépasser le stade traditionnel de la sensibilisation et assurer une continuité des démarches, de l'information préventive à la gestion réglementaire en passant par la négociation des choix d'acceptation des risques.

J. Grassin et G. GARRY [34] nous présentent une synthèse intéressante des travaux cartographiques qui ont été réalisés ces dernières années. L'exemple de l'atlas des zones inondables de la vallée de la Loire, qui n'aurait jamais vu le jour sans la volonté et l'implication personnelle permanente de J. GRASSIN, est aujourd'hui reconnu. La loi du 22 juillet 1987 sur l'information préventive a servi de support à cette initiative, qui traduit sa justification locale dans la nécessité de faire évoluer le débat et les références publiques concernant l'aménagement du bassin de la Loire et la maîtrise de l'urbanisation dans les zones inondables. Cette cartographie au $1 / 25000^{\mathrm{e}}$ présente les crues historiques connues, et un zonage de l'aléa centennal est organisé en 4 classes, selon la hauteur de submersion et la vitesse du courant.

C'est un modèle de référence, et les mesures gouvernementales de 1994 ont pu s'appuyer sur cette initiative pour la généraliser.

J. GRASSIN a été confronté à 2 difficultés méthodologiques :

- comment intégrer les modifications morphologiques qui ont introduit des modifications de lignes d'eau ?

- comment prendre en compte les risques de rupture des digues ?

Il a répondu partiellement à la première, considérant d'ailleurs à juste titre qu'il y avait urgence à restaurer des conditions d'écoulement aujourd'hui dégradées par défaut d'entretien. Cependant, les simulations hydrauliques qui ont servi d'appui à la cartographie tiennent compte des modifications majeures du lit. Pour les risques de rupture, une hypothèse statistique intégrant la connaissance de l'état actuel de ces ouvrages a été établie. Cette expérience concrète de porter à la connaissance de l'Etat, qui s'est en l'occurrence donné les moyens de ses objectifs, est exemplaire, en particulier parce qu'elle a bien permis de modifier les comportements des différents acteurs : il y a un « avant atlas » et un « après atlas ». M. GRASSIN insiste sur le fait que cette étape ne se fait pas sans mal, évidemment parce qu'elle dérange, et qu'elle a des impacts économiques et financiers non négligeables.

J. GRASSIN et G. GARRY citent également quelques autres initiatives. A la DDE de la Gironde, nous avions réalisé des études complètes des scénarios d'événements rares, intégrant les phénomènes complexes des marées, des 


\section{PRÉVENTION ET GESTION}

crues fluviales, et des surcotes liées à des phénomènes météorologiques (vent, pression atmosphérique). Cela avait conduit à définir, dans l'agglomération bordelaise, des scénarios de risques plus que centennaux, mais dont les conséquences sur une agglomération de 650000 habitants méritaient d'être mieux cernées. Ensuite, les débordements, dans leur dynamique heure par heure de ces scénarios ont été modélisés, afin d'obtenir une représentation réaliste des hauteurs de submersion. L'ensemble a été présenté au $1 / 25000^{c}$, en distinguant des classes mises au point avec la sécurité civile, et en intégrant les problèmes d'accessibilité aux zones sinistrées et les difficultés d'évacuation.

Cette étude est une première tentative pour développer un troisième type d'outil de cartographie, tourné, non plus seulement vers l'information préventive ou vers la maîtrise de l'urbanisation, mais vers l'identification des problèmes de secours et la recherche de solutions adaptées: on y reviendra dans la définition des politiques de prévention.

Dans les grands secteurs urbanisés, le problème de la cartographie, mais aussi tout simplement de la conservation d'une mémoire précise et quantifiée d'événements souvent localisés est une difficulté. C. COGEZ, I. PEREZ, F. Channeau, [35] en Seine-Saint-Denis, se sont attachés avec méthode à la constitution d'une base de données sur les zones inondées et les zones inondables, mise à disposition d'un très large public ; en effet, l'expérience leur a appris toute l'importance de s'appuyer sur des faits réels, des événements connus et répertoriés (zones inondées), pour faire percevoir aux divers acteurs économiques, aux aménageurs, l'importance des enjeux. Au délà de cette sensibilisation, il faut donner à tous ceux qui participent à l'acte de construire des règles simples, fondées sur l'appréciation des risques potentiels, bien qu'ils soient rarement perçus (zones inondables). La présence d'un urbaniste au sein de leur équipe, pour assurer ce dialogue avec les urbanistes, les architectes et les maîtres d'oeuvre, est certainement un atout essentiel.

En résumé :

- les démarches de cartographie du risque se sont développées de façon remarquable depuis 10 ans. Des produits tournés vers la sensibilisation et l'information préventive, vers la réglementation, vers la négociation d'une politique d'aménagement du territoire intégrant divers niveaux d'acceptation du risque, et enfin plus récemment vers l'analyse des problèmes de sécurité civile, se sont mis en place;

- la visualisation de la dynamique de la crue est un élément important dans la pédagogie, parce qu'elle permet une appropriation personnelle de mécanismes dont on n'a pas l'expérience vécue.

\subsection{Politiques de prévention}

B. de VAnSSAY [36] a réalisé des enquêtes psychosociologiques et économiques sur les 3 événements du GrandBornand (14 juillet 1987), de Nîmes (3 octobre 1988), et du Vaucluse (septembre 1992).

Rejoignant les remarques introductives du Président, elle constate que, contrairement aux idées reçues, il ne suffit pas de faire connaître le risque pour que la population adopte des comportements adaptés à la situation. L'action préventive doit donc se poser la question de savoir « quels sont les facteurs qui permettent aux individus (ou aux groupes) d'organiser, retenir et croire l'information qui leur est donnée... et de l'utiliser... ».

Il apparaît que l'expérience individuelle quotidienne est essentielle pour définir le comportement face à un risque exceptionnel. Ce n'est donc pas une information qu'il faut délivrer, mais un apprentissage quotidien et actif qu'il convient d'organiser.

La gestion de la post-crise est d'autre part extraordinairement sous-estimée: Mme de VANSSAY plaide pour l'élaboration de véritables «schémas directeurs de reconstruction " permettant d'intégrer à la fois les urgences, les transformations qu'il convient de préparer dans l'occupation des sols, et la définition des outils institutionnels nécessaires.

Enfin, Mme de VANSSAY nous présente une intéressante interprétation sociologique du retentissement médiatique et des représentations collectives qui s'installent à l'occasion de certains événements. Par exemple, à Nîmes, l'inondation a été vécue au travers d'un imaginaire collectif qui s'appuie sur une représentation des habitants d'appartenir à la "ville de la démesure ». Dans la phase de post-crise, quand circulent les rumeurs, les individus opèrent un travail de reconstitution et d'assimilation de l'événement dans le vécu individuel et collectif: c'est cette image reconstituée qui conditionne alors l'essentiel des comportements, plus que la réalité. Les événements des crues les plus récentes ne font que confirmer l'importance de cet " esprit des lieux » : la gestion de crise, le retentissement médiatique des ruptures des digues de Camargue, serait intéressant à comparer à ceux des crues dévastatrices du Nord-Est de la France dans la même période.

Deux études de cas peuvent servir d'introduction à la réflexion sur les politiques globales de prévention.

M. ThOMAS et P. GOMBERT [37] s'intéressent à un bassin très exposé, celui de l'Aude, dont un affluent, la Salz, a connu une crue dévastatrice, plus que centennale, estimée à $1000 \mathrm{~m}^{3} / \mathrm{s}$ pour un bassin-versant de $145 \mathrm{~km}^{2}$, quelques jours après la catastrophe de Vaison-la-Romaine, faisant 3 morts et $110 \mathrm{MF}$ de dégâts.

Une carte des zones inondées en 1992 a immédiatement été établie et diffusée à tous les maires pour être mise à disposition du public. L'aléa a été considéré comme fort dans la zone de grand écoulement, et dans les secteurs où la hauteur de submersion avait atteint $2 \mathrm{~m}$, ou atteindrait $1,5 \mathrm{~m}$ en centennal.

Des mesures de restriction de l'urbanisation ont été prises, et le dispositif d'annonce des crues de l'Aude a été étendu au bassin de la Salz. La rapidité des événements montre dans ce cas que les alarmes météorologiques seront extrêmement utiles.

J. de SaINT-Seine [38] nous présente le cas du Paillon à Nice. Il rappelle d'abord l'aménagement de ce petit fleuve côtier purement méditerranéen dont l'endiguement et la couverture dans la traversée de Nice ont démarré au XIXème siècle lors de l'accélération de la colonisation de sa plaine alluviale par l'urbanisation. Lors des derniers travaux, l'importance du transport solide a été prise en compte dans le dimensionnement. Un système d'alerte permet d'assurer dans des conditions de sécurité apparemment satisfaisantes la fermeture de la voie routière empruntant le lit du Paillon dans sa partie couverte sur $600 \mathrm{~m}$. Mais le propos de l'auteur aujourd'hui, à l'occasion des études conduites pour le P.E.R, est la nécessité de sensibiliser tous les acteurs autour de ce qu'il est possible de rencontrer pour une crue dépassant le centennal : même 
si cela peut apparaître un risque rare, l'ampleur des conséquences d'un tel événement dépassant l'évènement pris comme référence pour les projets suppose que l'on réfléchisse globalement à la façon de le gérer. L'auteur s'inquiète, dans ce domaine, du manque d'intercommunalité, et des développements qui se poursuivent sur l'amont du bassin et dont les conséquences peuvent être considérables.

J. NOYELLE [39] dresse un bilan des dix dernières années de la politique de prévention (depuis la loi de 1982 sur l'indemnisation des catastrophes naturelles) et décrit les nouvelles stratégies de prévention, telles qu'elles se dégagent des mesures gouvernementales de début 1994. De leur côté, J. GRASSIN et G. GARRY parviennent à des conclusions convergentes sur ce sujet. J. Noyelle insiste particulièrement sur une question, souvent posée par les services opérationnels, qui est celle du choix des aléas de référence. A travers les quelques exemples que nous avons cités, on peut reconnaître en effet un certain flou dans ce domaine. Les P.E.R. étaient établis en référence au décennal et au centennal pour les hauteurs d'eau et des vitesses d'écoulement se situant dans des fourchettes prédéfinies, mais relativement larges. Désormais, ces définitions sont assouplies en 3 niveaux de préoccupations :

- l'aléa courant ( 5 à 20 ans)

- l'aléa rare (50 à 200 ans)

- l'aléa exceptionnel (500 à 2000 ans) du type que nous avions évoqué pour Bordeaux, dans un esprit «étude de danger » proche des préoccupations de la sécurité civile.

J. NOYELLE remarque que la réponse aux enjeux liés à l'aléa courant se situe dans l'organisation des écoulements, intégrant une maîtrise en amont des eaux pluviales, et que les enjeux liés à l'aléa rare sont plutôt à prendre en compte à travers le droit des sols par la maîtrise de l'urbanisation, par la valorisation des zones d'expansion naturelle des crues, etc..., ceux de l'aléa exceptionnel relevant du strict domaine de la sécurité civile.

Il insiste sur la nécessaire complémentarité de l'approche à ces divers niveaux, dans un « projet de prévention ", dont l'élaboration pourrait être confiée aux CARIP (cellules d'analyse des risques et d'information préventive) prévues par circulaire du 13 décembre 1993 et qui se mettent progressivement en place dans tous les départements.

Mais il s'agit surtout, dans une réflexion du type des SAGE (schémas d'aménagement et de gestion des eaux) mis en place par la loi sur l'eau du 4 janvier 1992, d'assurer la cohérence entre la part de la gestion concertée (dont la méthode INONDABILITÉ peut être un outil) et celle du rôle réglementaire dont le Plan de Prévention des Risques sera l'outil.

Pour bien comprendre ces réflexions, nous allons faire une rapide analyse comparative des politiques engagées en 1982 et en 1994.

La politique mise en place en 1982 était fondée sur les principes et les mesures suivants :

- un principe de solidarité à l'égard des victimes de catastrophes naturelles traduit par un mécanisme d'assurance obligatoire ;

— une volonté de prévention exprimée par l'instauration d'un outil réglementaire, le PER, assurant une maitrise nouvelle de l'occupation des sols :
- dans les zones les plus exposées (rouges) la solidarité s'exerce vis-à-vis de l'existant, mais il y a plus de constructions nouvelles;

- dans les zones intermédiaires (bleues), la construction est possible sous réserve des prescriptions techniques, dont le non-respect implique la non-assurabilité du bien.

- Un souci de concertation se traduisant dans une procédure comportant de nombreux allers-retours entre l'Etat et les communes qui permettait en réalité aux communes qui le souhaitaient de bloquer le processus :

- un programme de travaux de protection hydraulique renforcé, concentré sur la protection de l'existant.

Cette démarche a indiscutablement été l'occasion d'une mobilisation autour des enjeux liés aux inondations et a stimulé les études et l'information à ce propos.

Cependant, plusieurs critiques lui ont été adressées :

- le volet «prévention " n'a pas eu l'efficacité du volet " solidarité », qui a dominé la mise en oeuvre. Il s'est organisé de ce fait un flux financier par l'assurance et l'indemnisation qui déresponsabilise l'acteur individuel ou la collectivité locale.

P. Bourgogne et D. Freziers [40] par exemple font remarquer qu'il n'a été envisagé dans le mécanisme d'assurance ni réduction des primes dans les zones non exposées, ni affectation d'une part du montant des primes au financement des travaux contribuant à réduire les risques, ni de réduction des primes après des travaux réduisant le risque. Ces auteurs montrent que les contribuables bordelais, qui ont financé des travaux de maîtrise des eaux pluviales (bassins de stockage et canalisations) considérables (3,5 MMF en 10 ans environ), sans aucune subvention de qui que ce soit, ont contribué à réduire très sensiblement la charge collective des catastrophes naturelles, sans obtenir de contrepartie de cet effort.

- Le principe de ne protéger, par des travaux, que l'existant, apparaît en réalité, difficile de mettre en œuvre. C'est ainsi que le Ministère de l'Environnement s'est trouvé financer des travaux de protection hydraulique de villes telles qu'Agen, où la part de la protection de nouveaux espaces pour en permettre l'urbanisation aura été non négligeable.

- Le souci de concertation, et le blocage qui semble en être résulté, procédait sans doute d'une confusion entre les divers niveaux de risques évoqués par J. NOYELLE, et d'un mélange peu clair entre les responsabilités de l'Etat et des collectivités.

- La coordination avec les S.A.E. (schéma d'aménagement des eaux, ancêtres des SAGE) qui existaient alors, n'était pas faite, et l'on retrouvait donc des politiques sectorielles « risques » et « eau » dont la cohérence n'était pas explicitée.

Les mesures nouvelles annoncées par le gouvernement en janvier et février 1994 ont l'ambition de répondre à ces divers enjeux :

- la protection de l'existant n'est plus un objectif en soi. Seuls quelques travaux ponctuels sont envisagés pour des cas très particuliers, et les travaux de restauration et d'entretien des cours d'eau sont en revanche relancés ;

- la recherche d'une conservation des zones d'expansion naturelle des crues, en y évitant les aménagements et en en maîtrisant l'urbanisation, devient une priorité. 
On constate par exemple dans les départements céréaliers comme l'Aube, que cette démarche est aujourd' hui largement facilitée par la jachère introduite par la politique agricole commune, de nombreux agriculteurs ayant choisi de mettre plutôt en jachère des terres inondables.

Des mécanismes financiers impliquant les agences de bassin, devraient permettre d'assurer une solidarité entre les bénéficiaires de cette protection par conservation des zones naturelles et ceux qui contribuent à les maintenir.

- Le plan de prévention des risques devient un cadre unique de l'action réglementaire, permettant aux services de l'Etat de traduire un niveau d'information même encore sommaire, en prescription, ce qui sera particulièrement utile pour les bassins-versants montagneux. A travers ce nouveau mécanisme, l'assurabilité des biens n'est plus le moteur principal de la réglementation. C'est en revanche le principe de la définition de niveaux de risques reconnus comme inacceptables, et de niveaux de risques compatibles avec les activités (le risque accepté) qui prédomine.

- La possibilité de relogement de personnes excessivement exposées devrait répondre à une attente de certains services opérationnels, confrontés parfois à des situations existantes.

H.P. WILLY, A. PETRASCHECK et J.P. JORDAN [41] nous présentent les mesures de la nouvelle loi fédérale sur l'aménagement des cours d'eau qui est entrée en vigueur en Suisse le $1^{\text {er }}$ janvier 1993.

Cette loi a pour but de trouver l'équilibre entre les besoins de protection d'un espace nécessaire aux activités humaines et la nécessité impérative de maintenir un cours et un régime naturel aux cours d'eau. La hiérarchie introduite dans les mesures rejoint clairement celles qui ont été initiées en France :

- d'abord l'entretien des cours d'eau et les mesures d'aménagement du territoire (interdiction de construire dans les zones à risques, prescription permettant une divagation suffisante des cours d'eau);

- éventuellement la correction des cours d'eau.

Par ailleurs sont définis les niveaux de protection que doivent respecter les divers types d'installation, en regard de la valeur des biens exposés. Enfin, l'examen de scénarios-catastrophes est envisagé. Ces divers éléments sont illustrés sur le bassin-versant de l'Engelberger Aa.

En résumé :

- l'évolution des politiques publiques en matière de prévention dans les 10 dernières années est forte, et semble partagée par divers pays européens. Il s'agit de parvenir à une synthèse véritable des politiques d'environnement et de prévention des risques:

- cette évolution suppose une intégration de ces enjeux dans l'aménagement du territoire. Cela n'est possible qu'à une échelle assez globale. Le morcellement des responsabilités communales en France est indéniablement un lourd handicap lorsqu'il s'agit de mettre en oeuvre ces principes ;

- la logique de solidarité, si elle n'est pas remise en cause, n'apparaît plus comme un principe unique suffisant. La nécessité de la responsabilisation de chacun, dans une logique de risque accepté, réapparaît plus activement aujourd'hui :

- l'information préventive ne saurait avoir de réelle efficacité si elle se limite à une communication sur l'existence d'un risque dont chacun ne se ferait pas une repré- sentation concrète. Cela suppose donc une appropriation des enjeux qui n'est pas faite aujourd'hui, et sans doute cela passe par une plus forte conscience de la responsabilité individuelle vis-à-vis de ces risques;

- la gestion post-crise est insuffisante, et devrait intégrer une logique de "schémas de reconstruction ";

- l'analyse des situations dépassant celles pour lesquelles les ouvrages ont été dimensionnés, est insuffisante.

\section{En conclusion}

Le dynamisme aujourd'hui de la politique de prévention des risques ne fait guère de doute à l'issue de ce tour de piste de différents domaines. Des progrès sensibles sont à portée de la main :

- développement du suivi et de la prévision hydrométéorologique à court terme :

- établissement pour les services d'annonce des crues d'outils standards de prévision éprouvés et d'utilisation pratique :

- préparation de systèmes-experts permettant de développer l'aptitude des prévisionnistes à apporter la meilleure plus-value en disposant d'éléments élaborés et adaptés à leurs besoins ;

- cartographies et simulations dynamiques adaptées aux diverses tâches de diagnostic rapide, de sensibilisation, du public, de réglementation et de préparation de crise ;

- volonté d'intégration de l'ensemble des actions dans une politique globale d'aménagement du territoire ;

- amélioration des plans de gestion post-crise.

\section{Références bibliographiques}

[1] P. КосH. - Cours d'hydrologie, ENPC, 1954».

[2] M. KerdoncuFF, A. MARIE et F. SEVAult. - « Valorisation de l'information des radars météorologiques », SHF 1994.

[3] F. BRESSAND et A. KAPFER. - « Apport des données radar de pluie pour la prévision et la gestion des crues rapides „ SHF 1994.

[4] D. Faure, H. Andrieu et J.D. Creutin. - * Comparaison d'estimations radar et pluviométriques de pluies horaires sur de petits bassins versants cévenoles », SHF 1994.

[5] J.J. Vidal, L. Bourrel, H. SAuVAgeot et J.P. Dupouyet. « Un nouvel outil pour l'annonce des crues: la télédétection par radar „, SHF 1994.

[6] G. Delrieu, R. Veyrier, M. Queau et D. Faure. * Caractérisation des précipitations méditerranéennes à de fines échelles spatio-temporelles », SHF 1994.

[7] P. Tourasse, R. Garcon, C. Obled, V. Aubonnet et C. DuFOUR. - « Nouveaux outils pour la prévision hydrométéorologique et le suivi des systèmes précipitants ", SHF 1994.

[8] P.A. Roche et al. - « Suivi et prévision des précipitations extrêmes ». Ministère de l'Environnement. 1990.

[9] P.A. RochE et al. - * Les crues et laves torrentielles Prévention et gestion, actes du colloque des 24 au 26 juin 1992 à Nainville-lès-Roches », SHF 1992, Paris.

[10] J.D. Creutin, H. ANdrieu et W. Krajewski. - $*$ Prévision à très courte échéance des intensités pluvieuses: utilisation des informations des radars météorologiques ». SHF 1994. 
[11] H. ANDrieu, V. Thauvin et M.N. French. $-\approx$ Application d'un modèle de prévision de pluie à très courte échéance au contexte cévenol », SHF 1994.

[12] C. Godon et M. OdIER. - $\propto$ L'annonce des crues : une des priorités du Ministère de l'Environnement en matière d'inondations „, SHF 1994.

[13] L. Moulin et D. REINBolD. - " Le réseau de collecte de données hydrométéorologiques du bassin de la Loire : bilan de l'exploitation et enseignement sur la fiabilité », SHF 1994.

[14] C. LAllement et P. Tourasse. - " Comportement et utilisation opérationnelle d'un réseau hydroclimatologique en période de crise ». SHF 1994.

[15] J.L. RoY. - « Annonce des crues. Avantages et inconvénients du système en chaîne. Proposition pour un renforcement $»$, SHF 1994.

[16] A.P. Boulllot, B. Voron et M. Mel.KI. - « Réseau d'observation hydrologique et hydraulique de la Ville de Nîmes », SHF 1994.

[17] C. Obled, J.Y. Rodriguez et G.M. Saulnier. - * Une approche opérationnelle pour la modélisation des bassins versants sujets aux crues éclair », SHF 1994.

[18] P.A. Roche et al. - «Guide de prévision des crues », SHF 1987.

[19] X. YANG, C. MiChel et P.A. Roche. - "Une méthode d'adaptation d'un modèle conceptuel pluie-débit pour la prévision des crues en temps réel », SHF 1994.

[20] P.A. Roche et al. - $\propto$ Propositions pour une intercomparaison de modèles de prévision de crues », SHF 1991.

[21] D. Duband, J.L. Nègre, J. Cunge et A. Kapfer, - "Une méthodologie et un système intégré de prévision de crueséclairs, pour les services d'annonces des crues „, SHF 1994.

[22] P.A. RochE et R. TASSIN. — " La combinaison de modèles, un moyen de limiter l'impact des perturbations en prévision des crues. Revue internationale des sciences de l'eau „Vol. 3 , $n^{\circ} 2$, mai 1987, 57-65.

[23] J.P. DuPOUYeT, P. COHEN, C. Feliu, P. Glize et J.J. VidAL. — "Projet "SEC" - Système multi-Expert pour la prévision des Crues - Bassin de la Garonne », SHF 1994.

[24] C. ThIRriot et H. HABAIEB. - "Comparaison de simulation de prévision manuelle de crue par des groupes d'opérateurs de nationalités et d'expériences différentes „. SHF 1994.

N.B. : les références « SHF 1994 » renvoient aux actes du colloque "crues et inondations " de la SHF.
[25] J.L. RAHUEl, J.L. NËgRE et J.A. CUNGE. - SHANGHAl : Gestion prévisionnelle des inondations „, SHF 1994.

[26] J.M. Delattre, G. Auriaux et P. Voignier. - « Evolution de la gestion des risques hydrauliques sur un réseau d'assainissement. L'expérience de la Seine Saint-Denis », SHF 1994.

[27] D. FrÉsIERS et P. BOURGOGNE. - - RAMSES : un télécontrôle de crise $\$$, SHF 1994.

[28] X. CAIlmall et P. LE Delliou. - « Elaboration des consignes de crues. Prise en compte des situations exceptionnelles $\$$, SHF 1994.

[29] A. Faure-Soulet, M. Masson, J. Lavabre, P. Givone et O. GILARD. - *Identification et évaluation sommaire des risques d'inondations liées au ruissellement périurbain et aux crues torrentielles », SHF 1994.

[30] B. Chastan, O. Gilard, P. Givone et G. Oberlin. - $~$ Le modèle inondabilité, présent et avenir ", SHF 1994.

[31] O. Barozet, O. Gillard, P. Givone et M. SOMmier. - $*$ Le modèle de gestion hydraulique du bassin versant de la Bourbre $\$$. SHF 1994.

[32] J.N. GAutier et L. GAZulL. - «Inondabilité : de la recherche au bureau d'étude s, SHF 1994.

[33] H. DUMAY. - * CALYPSEAU: outil d'identification et de prévision du risque d'inondation par interprétation cartographique $\gg$ SHF 1994.

[34] G. Garry et J. Grassin. - * La prise en compte du risque d'inondation dans l'urbanisme et le droit des sols». SHF 1994.

[35] C. Cogez, I. Perez et F. Chaumeau. - $*$ Le risque d'inondation en ville lors de fortes pluies: le situer, le quantifier, le faire connaître », SHF 1994.

[36] B. de VANSSAY. - Information, prévention et reconstruction „: " si vis pacem, para bellum », SHF 1994.

[37] M. ThOMAs et P. GOMBERT. - Q Quelles mesures préventives pour les bassins versants ruraux à réponse rapide? », SHF 1994.

[38] J. de SAINT-SEINE. - - La gestion du risque inondation : le cas du Paillon à Nice », SHF 1994.

[39] J. NOYELLE. - * L'évolution des stratégies de prévention contre les inondations „. SHF 1994.

[40] P. BOURGONE et D. FrésIERs. - - Assurances tous risques $\gg$, SHF 1994.

[41] H.P. Willi. A. PEtrascheck et J.P. Jordan. - * Nouvelle orientation dans le domaine de la protection contre les crues en Suisse. Recommandations et applications pratiques „, SHF 1994. 